\title{
Multiexpandable cage for minimally invasive posterior lumbar interbody fusion
}

\author{
This article was published in the following Dove Press journal: \\ Medical Devices: Evidence and Research \\ 28 September 2016 \\ Number of times this article has been viewed
}

\author{
Jeffrey D Coe' \\ James F Zucherman ${ }^{2}$ \\ Donald W Kucharzyk ${ }^{3}$ \\ Kornelis A Poelstra ${ }^{4}$ \\ Larry E Miller ${ }^{5}$ \\ Sandeep Kunwar ${ }^{6}$ \\ 'Silicon Valley Spine Institute, \\ Campbell, ${ }^{2}$ San Francisco Orthopaedic \\ Surgeons, San Francisco, CA, \\ ${ }^{3}$ Orthopaedic Pediatric and Spine, \\ Crown Point, IN, ${ }^{4}$ Department of \\ Surgery, Sacred Heart Hospital on \\ the Emerald Coast, Miramar Beach, \\ FL, ${ }^{5}$ Miller Scientific Consulting, Inc., \\ Asheville, NC, ${ }^{6}$ Bell Neuroscience \\ Institute, Washington Hospital \\ Healthcare System, Fremont, CA, USA
}

Correspondence: Jeffrey D Coe Silicon Valley Spine Institute, 22I East Hacienda Avenue, Suite A, Campbell, CA 95008, USA

Email jcoe@svspine.com

\begin{abstract}
The increasing adoption of minimally invasive techniques for spine surgery in recent years has led to significant advancements in instrumentation for lumbar interbody fusion. Percutaneous pedicle screw fixation is now a mature technology, but the role of expandable cages is still evolving. The capability to deliver a multiexpandable interbody cage with a large footprint through a narrow surgical cannula represents a significant advancement in spinal surgery technology. The purpose of this report is to describe a multiexpandable lumbar interbody fusion cage, including implant characteristics, intended use, surgical technique, preclinical testing, and early clinical experience. Results to date suggest that the multiexpandable cage allows a less invasive approach to posterior/transforaminal lumbar interbody fusion surgery by minimizing iatrogenic risks associated with static or vertically expanding interbody prostheses while providing immediate vertebral height restoration, restoration of anatomic alignment, and excellent early-term clinical results.
\end{abstract}

Keywords: degenerative disc disease, expandable, low back pain, Luna

\section{Introduction}

Low back pain (LBP) is one of the most common reasons for physician visits and hospitalizations worldwide. ${ }^{1}$ Although LBP typically resolves spontaneously, $10 \%$ of the adult population suffers from chronic LBP. The clinical and economic burden of persistent LBP is enormous, resulting in significant disability, lost work days, and excessive health care resource utilization. ${ }^{2}$ Degeneration of the lumbar intervertebral discs is common with advancing age ${ }^{3,4}$ and represents the primary cause of chronic LBP in adults. ${ }^{5}$ Nonsurgical treatments such as physical therapy, analgesics, and activity modification are the first-line therapies for LBP secondary to degenerative disc disease (DDD). However, patient prognosis with nonsurgical treatments is poor when symptoms persist for 6 months or more. ${ }^{6-9}$

In patients with chronic disabling LBP unresponsive to nonsurgical therapies, or those who develop instability in the process of decompressing neural compression, lumbar interbody fusion with rigid fixation is a surgical option that removes pain-generating compressive tissue, eliminates painful segmental motion, and restores sagittal balance..$^{10}$ Traditional surgical access corridors for lumbar spine fusion include anterior lumbar interbody fusion (ALIF), lateral lumbar interbody fusion (LLIF), transforaminal lumbar interbody fusion (TLIF), and posterior lumbar interbody fusion (PLIF). Each of these access corridors is associated with a distinct risk profile, depending on the 
anatomic structures that must be traversed in order to gain access to the index interspace. ${ }^{11}$

Anterior approaches (ALIF, LLIF) have highly successful clinical outcomes, due in part, to the better access and the larger-sized implants that can be used. However, use of an anterior approach is often stymied by anatomic restraints such as obesity and encumbrance of the iliac crest for accessing L5-S1, and neural structures at L4-L5 for LLIF. Posterior approaches, particularly TLIF, have been used with consistently higher frequency over the last decade as a means to avoid the significant iatrogenic vessel and other injuries associated with ALIF and overcome other barriers to anterior accessibility while providing the ability for direct neural decompression. The surgical corridor used with posterior (PLIF and TLIF) approaches, particularly minimally invasive approaches, is necessarily narrow due to the proximity of neural structures. Risks of posterior spinal fusion include injuries associated with nerve root retraction and manipulation of the dura, and iatrogenic injuries related to impacted insertion of an interbody cage, especially in the presence of spondylolisthesis, lumbar lordosis, and disc collapse. ${ }^{12,13}$

Vertically expanding interbody cages were introduced in recent years as a way to overcome the obstacles of a narrow posterior surgical corridor. Vertically expanding cages are designed to be inserted into the disc space in a collapsed configuration and then their height is expanded in situ to restore disc height. The size of an interbody cage that may be used in a posterior approach is constrained, which may negatively impact the ability to place sufficient bone graft into the surgical space and increase the potential risks of subsidence, biomechanical instability, and surgical failure. ${ }^{14-16}$ A multidimensional expandable large footprint interbody device may be helpful for addressing the current shortcomings of minimally invasive PLIF surgery. The purpose of this report is to describe a recently introduced multiexpandable lumbar interbody fusion cage designed to be used in posterior approach fusion surgery. Its technical features, surgical technique, preclinical testing results, and early clinical experience are discussed.

\section{Device}

The multiexpandable interbody cage described here (Luna $3 \mathrm{D}^{\circledR}$ Interbody Fusion System, Benvenue Medical Inc., Santa Clara, CA, USA) comprises three interlocking polyetheretherketone components, a lock wire and screw, and a graft window that allows bone graft material to be inserted into the cage after in situ expansion (Figure 1). The cage is delivered from a rectangular cannula that ranges between 6 and $8 \mathrm{~mm}$ in height and width, depending upon the size of the cage to be used. The Luna $3 \mathrm{D}$ cage is delivered in two separate stages. First, the top and bottom components (between 5 and $8 \mathrm{~mm}$ in height) are simultaneously inserted into the disc space; as they are progressively inserted, they curl back upon themselves into a circular configuration of $\sim 25 \mathrm{~mm}$ in diameter. Next, the middle component is advanced between the top and bottom components, increasing the height of the cage. A cage height from 8 to $15 \mathrm{~mm}$ may be selected by the surgeon, depending on the patient's anatomy. The method used to insert the cage allows minimal-impaction delivery, which protects and preserves the vertebral endplates. One of the main advantages of a multiexpandable cage is that an ALIF-like cage-sized footprint (Figure 2) can be delivered through a minimally invasive, narrow posterior surgical corridor.

\section{Procedure}

Once the patient has been managed as usual for spinal fusion surgery and access to the index interspace is achieved, a standard discectomy is performed. The discectomy should consist of thorough removal of the nucleus, whereby the cortical endplate bone is maximally exposed to enhance apposition of the endplates with the Luna 3D cage and bone
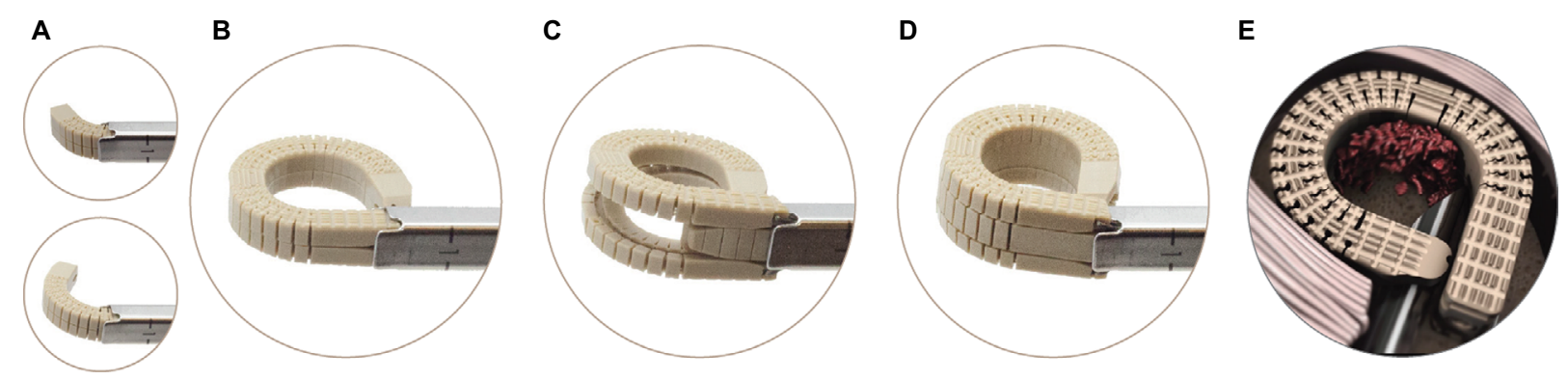

Figure I Luna 3D ${ }^{\circledR}$ (Benvenue Medical Inc., Santa Clara, CA, USA) lumbar interbody fusion cage.

Notes: Major steps of deployment include (from left to right): (A) initial deployment of top and bottom components in a curved trajectory from a cannula (top and bottom images), (B) completion of top and bottom component deployment in a circular configuration, (C) initial advancement of middle component between top and bottom components, (D) completion of Luna 3D cage deployment, and (E) bone graft injection into implant concavity. 


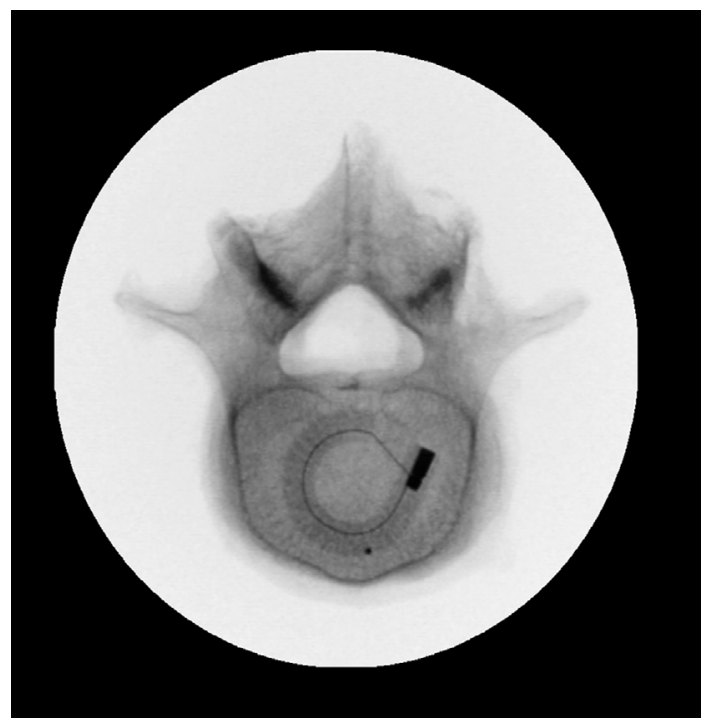

Figure 2 Axial view of Luna 3D (Benvenue Medical Inc., Santa Clara, CA, USA) lumbar interbody fusion cage in situ - showing an ALIF cage-type footprint. Abbreviation: ALIF, anterior lumbar interbody fusion.

graft to promote fusion of the target motion segment. Next, height of the prepared disc space is carefully measured with a sizing paddle to determine the appropriate-sized cage to place into the disc space. As an available option, a Luna 3D trial device may be utilized before placing the Luna 3D cage as a means to confirm disc space height measurements obtained with the sizing paddle and to ensure that sufficient discectomy was performed.

For deployment of the Luna 3D cage, the top and bottom components of the cage are deployed from the cannula into the disc space in stacked configuration (Figure 3). Correct positioning of the cage is confirmed by assessing radiopaque markers on the cage under fluoroscopy. After insertion, the location of the cage is confirmed, paying particular attention to the cage fit at the posterior aspect of the disc space using a lateral radiographic view and the central orientation of the cage on the midline of the disc space using an anteroposterior view. The middle component of the cage is then advanced between the top and bottom components until its radiopaque marker is aligned with those of the top and bottom components. With insertion of the middle component, in situ distraction (elevation) of the endplates is achieved and intervertebral disc height is restored.

Following full deployment of the cage, the device handle and deployment cables are removed. Bone graft is injected into the center of the cage through a small window; this allows the graft to be optimally packed into the intervertebral space, facilitating complete endplate to endplate bone graft bridging. The cage is then locked into place using the internal lock wire
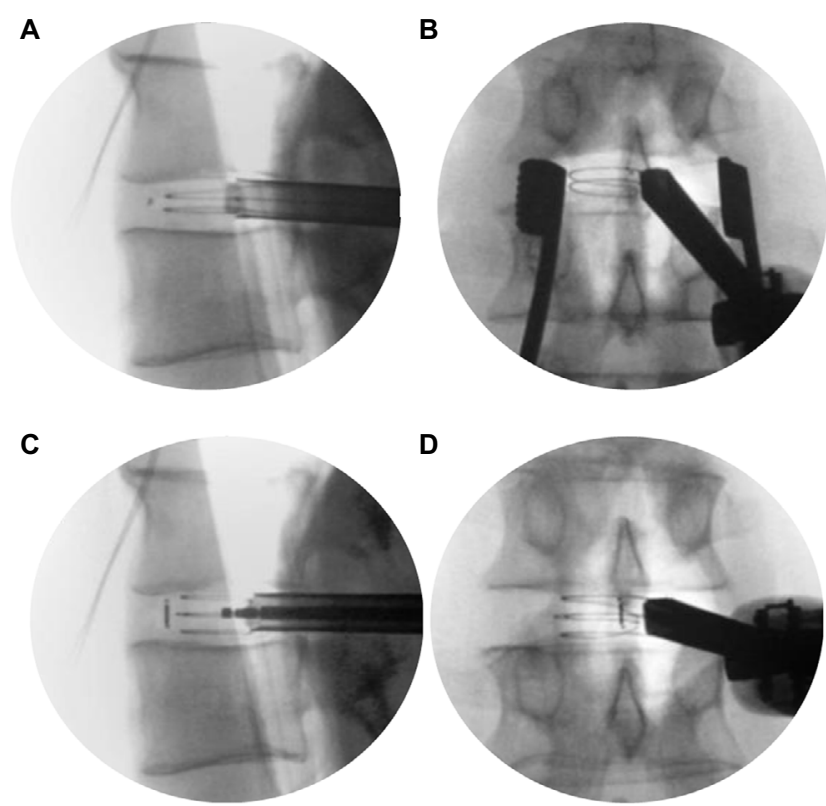

E
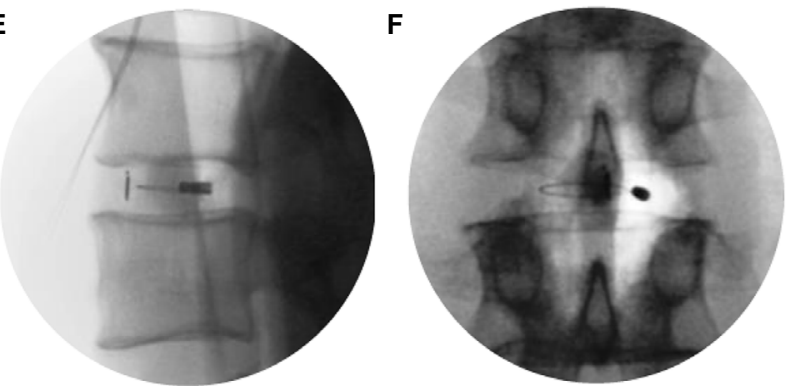

Figure 3 Lateral (A) and anteroposterior (B) radiographic views of initia deployment, middle component deployment (C and $\mathbf{D})$, and fully deployed Luna $3 D^{\circledast}$ (Benvenue Medical Inc., Santa Clara, CA, USA) lumbar interbody fusion cage ( $E$ and $F$ ).

and screw, which provides a stable construct. Implant stability is further enhanced by superior and inferior teeth that enhance resistance to migration. Supplemental posterior fixation, most commonly bilateral pedicular screws, is then applied.

\section{Preclinical testing}

Extensive preclinical testing was performed with the Luna 3D cage to confirm safety, performance, durability, and resistance to compression and torsion (Benvenue Medical Inc, unpublished data, 2014). Per US Food and Drug Administration guidance for interbody devices, Luna was subjected to a variety of static and dynamic mechanical testing to demonstrate that it performs equivalent or better to currently marketed devices per American Society for Testing and Materials 2267. Static mechanical testing in compression, compression-shear, and torsional modes showed the cage yield strength to be well above those of the intervertebral disc and vertebral body. Luna exhibits a static axial yield of $>23 \mathrm{kN}$, static torsional yield of $>10.0 \mathrm{Nm}$, and compression-shear yield of $>4.4 \mathrm{kN}$. Dynamic 
mechanical testing up to 5 million cycles demonstrated the ability to withstand axial loading to $3 \mathrm{kN}$, torsional loading to $>2 \mathrm{Nm}$, and compression-shear loading to $>1.5 \mathrm{kN}$. Additionally, no cage failures were identified even at FDAmandated supraphysiologic loads. Resistance to subsidence was excellent, with an average subsidence stiffness equaling or surpassing the values of competitive predicate cages.

Validation of the surgical procedure used to place the cage was conducted in human cadaver specimens. One hundred percent procedural success was achieved with multiple surgeons performing multilevel deployments in six unique human cadaver specimens. The cage was also confirmed to be compatible with magnetic resonance imaging up to $3 \mathrm{~T}$. The Luna 3D cage received FDA 510k clearance in November 2014. The FDA judged the cage to be substantially equivalent to already marketed predicate devices, suggesting safe and durable structural support in patients undergoing lumbar interbody fusion for chronic lumbar DDD.

Subsequently, a biomechanical study was performed by Voronov et al from Loyola University in Chicago to demonstrate equivalence in biomechanical stability between the Luna 3D cage implanted via a posterior TLIF approach and a typical ALIF cage implanted using an ALIF approach. ${ }^{17}$ Twelve fresh frozen cadaveric spine specimens (L1-L5) with a mean age of $47.5 \pm 10.6$ years were instrumented using one of five study constructs: 1) intact spine; 2) ALIF approach with stand-alone ALIF cage; 3) ALIF approach with ALIF cage + bilateral pedicle screws; 4) TLIF approach with stand-alone three-dimensional expandable cage; and 5) TLIF approach with three-dimensional expandable cage + bilateral pedicle screws.

The biomechanical stability of the Luna 3D cage placed via TLIF was concluded to be equivalent to an ALIF cage placed via ALIF, both when used as stand-alone devices as well as when supplemented with bilateral pedicle screw-rod stabilization. The two devices significantly reduced lumbar segmental motion at the level of placement, and there was no statistical difference between the surgical constructs in reducing flexion-extension with preload, lateral bending, or axial rotation. Statistical difference was observed under flexion-extension without preload, where the Luna 3D cage construct produced a greater reduction in motion compared to the ALIF construct.

\section{Early clinical experience}

A multicenter medical chart review study intended to evaluate outcomes of a multicenter series of patients undergoing TLIF or PLIF with the Luna 3D cage was initiated by the authors and is currently ongoing. This research received Western Institutional Review Board approval, and a waiver of consent was granted. It was hypothesized that patients would demonstrate decreased pain, improved function, and improved radiological findings, including restored foraminal height, improved disc height, and lordotic angle after the fusion surgery. To date, 32 patients (20 males, ages 24-82 years) with clinical follow-up at 3 months are included in the data analysis (Table 1). Primary diagnoses include spinal stenosis, spondylolisthesis, radiculopathy, DDD, and scoliosis. Half of the current patient cohort had previous spine surgery.

Using a minimally invasive, mini-open, or open surgical approach, as appropriate, 17 patients had a single-level procedure and 15 patients had a multilevel procedure (Table 2). In the multilevel procedures, the Luna 3D cage was placed at one or two levels, and in some cases along with a static cage at other contiguous level(s) between L2-L3 and L5-S1, which was placed using either a TLIF or LLIF approach.

Clinical results of the study cohort are summarized in Table 3. No neurologic complications were seen. One patient had a small dural tear without cerebrospinal fluid leak at a Luna 3D level, which required no repair. At 3 months, radiculopathy was resolved in $21 / 22$, neurologic deficit in $16 / 20$ (+2 improving), and motor deficit in 12/15 (+2 improving) patients. Back and leg pain were reduced, with minimal

Table I Baseline patient characteristics $(n=32)$

\begin{tabular}{ll}
\hline Variable & Value \\
\hline Demographics & \\
Age, years ${ }^{a}$ & $59 \pm 16$ \\
Male sex & $63 \%$ \\
Diagnosis & \\
Spinal stenosis & $72 \%$ \\
Spondylosis & $66 \%$ \\
Spondylolisthesis & $56 \%$ \\
Herniated nucleus pulposus & $44 \%$ \\
Degenerative disc disease & $34 \%$ \\
Scoliosis & $9 \%$ \\
Symptoms & \\
Radiculopathy & $69 \%$ \\
Neurological deficits & $63 \%$ \\
Motor deficits & $47 \%$ \\
Pain & \\
Back and leg & $84 \%$ \\
Back only & $3 \%$ \\
Leg only & $13 \%$ \\
Prior spine surgery & \\
Any spine surgery & $50 \%$ \\
Laminectomy & $31 \%$ \\
Discectomy & $9 \%$ \\
Fusion & $28 \%$ \\
\hline Note: &
\end{tabular}

Note: ${ }^{a}$ Mean \pm standard deviation. 
Table 2 Operative data

\begin{tabular}{ll}
\hline Variable & Value \\
\hline Surgical approach & 17 \\
Minimally invasive & 2 \\
Mini-open & 13 \\
Open & 17 \\
Single-level posterior procedure (TLIF or PLIF) & 9 \\
L5-SI & 6 \\
L4-L5 & 1 \\
L3-L4 & 1 \\
L2-L3 & 15 \\
Multilevel procedure & 1 \\
TLIF with Luna 3D ${ }^{\circledR}$ (Benvenue Medical Inc., & \\
Santa Clara, CA, USA) cage & 7 \\
TLIF with Luna 3D cage (one level), static cage & \\
additional level(s) & 7 \\
TLIF with Luna 3D cage (one level), LLIF & \\
with static cage additional level(s) & 35 \\
Number of levels with Luna 3D cage & 21 \\
L5-SI & $1 \mathrm{I}$ \\
L4-L5 & 2 \\
L3-L4 & 1 \\
L2-L3
\end{tabular}

Abbreviations: LLIF, lateral lumbar interbody fusion; PLIF, posterior lumbar interbody fusion; TLIF, transforaminal lumbar interbody fusion.

Table 3 Clinical outcomes at 3 months $(n=32)$

\begin{tabular}{ll}
\hline Variable & Value \\
\hline Symptoms resolved & \\
Radiculopathy & $21 / 22$ \\
Neurologic deficit & $16 / 20^{\mathrm{a}}$ \\
Motor deficits & $12 / 15^{\mathrm{a}}$ \\
Pain reduction / function improvement & \\
Back pain - MCID & $27 / 29(93 \%)$ \\
Leg pain - MCID & $27 / 29(93 \%)$ \\
ODI - >I2 points improved & $13 / 18(72 \%)$ \\
X-ray measure improvementsc, d & \\
Disc height & $4.5 \pm 2.5$ \\
Local disc angle & $6.1 \pm 3.4$ \\
Foraminal height & $5.6 \pm 3.7$ \\
Regional lumbar lordosis & $1.6 \pm 3.7$ \\
\hline
\end{tabular}

Notes: a'Two additional patients were reported as "improving"; bdata not available for all patients; ${ }^{c} n=29$ levels; ${ }^{d}$ mean \pm standard deviation.

Abbreviations: MCID, minimal clinically important difference; ODI, Oswestry Disability Index.

clinically important difference (MCID) achieved by $>90 \%$ of patients. The MCID for pain in this study included medical chart notations of "no pain", "minimal pain", or "reduced pain". In 18 patients with available Oswestry Disability Index (ODI) scores, 13 (72\%) were improved $\geq 12$ points, which is considered MCID for ODI. ${ }^{18}$ For 29 levels with the Luna 3D cage, X-ray measures at 3 months showed median (range) improvements of 6 ( -1 to 14$)$ degrees for local disc angle, 4 (0 to 9.5) $\mathrm{mm}$ for average disc height, and 5 (-1 to 14$) \mathrm{mm}$ for foraminal height at the Luna $3 \mathrm{D}$ level. One site obtained computed tomography scans on four patients who had a single-level procedure with the Luna 3D cage. Regional lumbar lordosis improvements of 4, 6, 12, and 16 degrees were seen. No evidence of subsidence or migration was noted on X-rays or computed tomography scans in any case and several patients showed radiologic signs of fusion at 3 months. Early clinical experience is demonstrating encouraging outcomes, including absence of nerve retraction injuries as well as restoration of disc height and restoration or preservation of lordosis.

Representative clinical and radiographic outcomes with the multiexpandable cage are highlighted with one case from the series.

\section{Case}

A 73-year-old female presented with a 5-year history of bilateral L5 radicular pain following unsuccessful nonsurgical treatment. Nonoperative treatment had included physical therapy, activity modification, medications, and lumbar epidural steroid injections, which were helpful for several years initially but the most recent set had only provided 3-4 weeks of pain relief. Pain severity was $9 / 10$ at its peak and was present almost all the time. Her preoperative ODI score was 30. Imaging studies revealed grade I-II degenerative spondylolisthesis at L4-5, severe spinal stenosis, and total collapse of the disc space (Figure 4). She underwent minimally invasive TLIF with a $9 \mathrm{~mm}$ height Luna 3D cage using local and allograft bone grafts with bilateral pedicle screw fixation. Procedural blood loss was minimal (100 cc), and the patient was discharged 2 days after surgery. On postoperative day 11 , she presented to the emergency room after a fall. Her computed tomography scan was negative and she was discharged home. At the 3-month follow-up visit, her LBP and lower extremity pain had completely resolved ( $0 / 10$ for both), ODI score was 0 , and radiological findings showed a stable construct with restoration of the L4-L5 disc space (Figure 5).

\section{Discussion}

The increasing adoption of minimally invasive techniques for spine surgery in recent years has led to significant advancements in instrumentation for lumbar interbody fusion. The capacity to deliver a multiexpandable interbody cage with a large footprint through a narrow surgical cannula represents a significant advancement in spinal surgery technology. Percutaneous pedicle screw fixation is now a mature technology, but the role of expandable cages has not been heretofore welldefined. Although their role is often critical for achieving a successful fusion, PLIF/TLIF cage technology, to date, has 

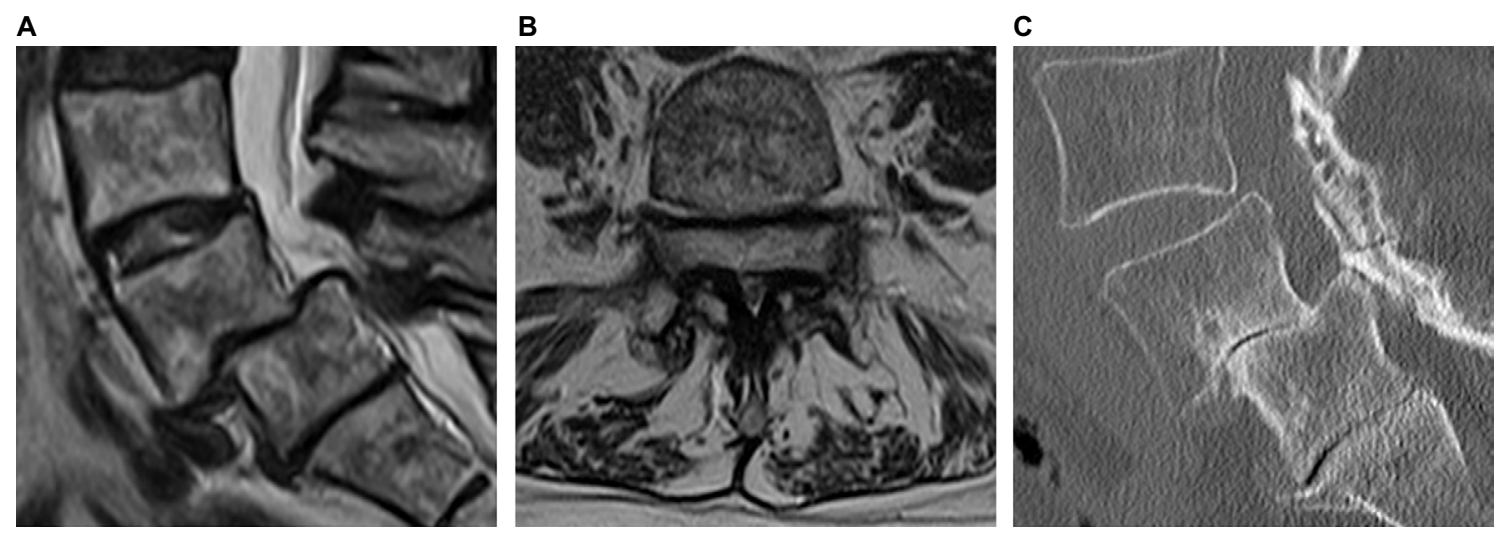

Figure 4 Sagittal (A) and axial (B) MRI and sagittal CT (C) scans show grade I-II degenerative spondylolisthesis at L4-L5, total collapse of the disc, and severe spinal stenosis. Abbreviations: $\mathrm{CT}$, computed tomography; MRI, magnetic resonance imaging.
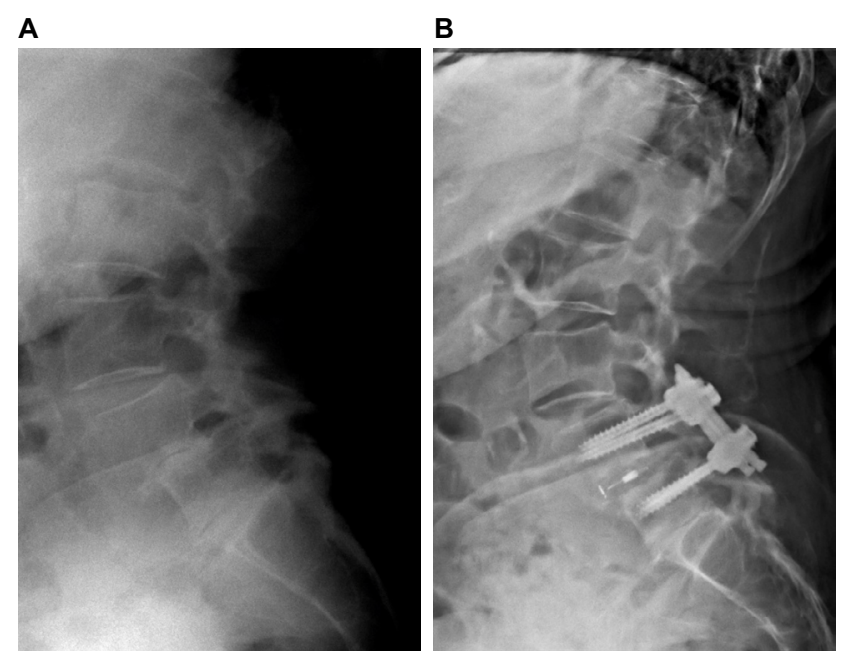

Figure 5 Radiographs showing preoperative grade I-II degenerative spondylolisthesis with total disc collapse at L4-5 (A) and stable construct with restoration of disc space 3 months after minimally invasive TLIF with Luna 3D ${ }^{\circledR}$ (Benvenue Medical Inc., Santa Clara, CA, USA) cage (B). At 3 months, the patient is doing well clinically and reported complete resolution of low back and lower extremity pain.

Abbreviation: TLIF, transforaminal lumbar interbody fusion.

not been developed adequately to be included in the truly minimally invasive surgery toolbox.

The ability to deliver an ALIF cage-sized footprint using a minimally invasive posterior approach has obvious advantages, including eliminating the need for an ALIF access surgeon and avoiding ALIF approach-related risk of injury. The design of the Luna 3D cage permits it to engage broader, peripheral sections of the vertebral endplates, whereas most posterior interbody cages typically engage smaller, more central portions of the end plate. This is advantageous since patients with DDD have a greater risk of subsidence when a cage with a smaller footprint is placed in the central or posterior endplate regions. ${ }^{19,20}$ A multiexpandable cage allows a larger footprint to be obtained than that obtained with a conventional posterior cage, without the need for making a larger incision or having to perform greater nerve root retraction. Furthermore, unlike many other expandable interbody cages, the multiexpandable cage described herein is radiolucent, which allows accurate radiographic assessment of fusion. Finally, the geometry of this multiexpandable cage allows for a large volume of contiguous bone graft to be placed post expansion, which is likely to optimize the local environment for successful vertebral body fusion.

Thorough patient evaluation and selection along with careful attention to proper surgical technique are key factors to achieving excellent outcomes. Patients with active infection, metabolic bone disease, malignancy, high-grade spondylolisthesis, morbid obesity, or DDD affecting three or more levels may be poor candidates for this procedure. Importantly, minimally invasive lumbar interbody fusion may prove to be a technically more demanding procedure since the operator is working through a narrow surgical corridor with limited visualization of anatomic landmarks. Identification of proper placement of the cage under fluoroscopy is essential to confirm proper implant position. In this case, the cage components may be retracted and repositioned prior to expansion until the desired location is achieved.

As with any interbody cage, another source of potential concern is the need for future revision surgery. With this multiexpandable cage, removal is performed in the opposite manner as the initial deployment using the same approach. Briefly, the cage is unlocked, the middle component is removed first, followed by removal of the stacked top and bottom components simultaneously. Spine surgeons must be familiar with relevant anatomy, undergo training and experience with the approach, and be adept at prevention, recognition, and treatment of potential complications in order to achieve optimal clinical results. Areas of future research include procedural outcome comparisons to static and vertically expandable interbody cages, evaluation of longer-term clinical and radiographic outcomes, and cost utility assessments. 
In summary, the multiexpandable cage described here allows a far less invasive approach for performing PLIF procedures by minimizing iatrogenic risks of impacted insertion of smaller footprint cages while providing an ALIF cage-type footprint and immediate vertebral height restoration, anatomic alignment, and excellent early clinical results.

\section{Disclosure}

This study was supported by Benvenue Medical, Inc.

JDC is a consultant to Benvenue, Nuvasive, Providence Medical Technology, and SI Bone; receives research support from Benvenue, Medtronic Sofamor Danek, Nuvasive, and SI Bone; serves on the board of California Orthopedic Association, Cervical Spine Research Society, Lumbar Spine Research Society, and Scoliosis Research Society; and stock ownership in Alphatec Spine, Benvenue, and Phygen. JFZ reports no conflicts of interest. DWK is a consultant to Benvenue, Biomet/Zimmer, Medicrea, Precision Spine, Orthofix, and SeaSpine; receives research support from Benvenue, receives royalties from Precision and Medicrea, serves on the surgical advisory board for Precision, and serves on the speaker's bureau for Benvenue, Precision, Biomet/Zimmer, Medicrea, Orthofix, SeaSpine. KAP is a consultant to Benvenue, K2M, MiRus, Mazor Robotics, DePuy Synthes Spine, and ISD, and reports patent coownership in K2M and DePuy Synthes Spine. LEM is a consultant to Benvenue. SK is a consultant to Benvenue. The authors report no other conflicts in this work.

\section{References}

1. Balague F, Mannion AF, Pellise F, Cedraschi C. Non-specific low back pain. Lancet. 2012;379(9814):482-491.

2. Parthan A, Evans CJ, Le K. Chronic low back pain: epidemiology, economic burden and patient-reported outcomes in the USA. Expert Rev Pharmacoecon Outcomes Res. 2006;6(3):359-369.

3. Niu G, Yang J, Wang R, Dang S, Wu EX, Guo Y. MR imaging assessment of lumbar intervertebral disk degeneration and age-related changes: apparent diffusion coefficient versus T2 quantitation. AJNR Am J Neuroradiol. 2011;32(9):1617-1623.

4. Hicks GE, Morone N, Weiner DK. Degenerative lumbar disc and facet disease in older adults: prevalence and clinical correlates. Spine (Phila Pa 1976). 2009;34(12):1301-1306.
5. DePalma MJ, Ketchum JM, Saullo T. What is the source of chronic low back pain and does age play a role? Pain Med. 2011;12(2): 224-233.

6. Chou R, Atlas SJ, Stanos SP, Rosenquist RW. Nonsurgical interventional therapies for low back pain: a review of the evidence for an American Pain Society clinical practice guideline. Spine (Phila Pa 1976). 2009;34(10):1078-1093.

7. Carey TS, Garrett JM, Jackman AM. Beyond the good prognosis. Examination of an inception cohort of patients with chronic low back pain. Spine (Phila Pa 1976). 2000;25(1):115-120.

8. Von Korff M. Studying the natural history of back pain. Spine (Phila Pa 1976). 1994;19(18 Suppl):2041S-2046S.

9. Smith SE, Darden BV, Rhyne AL, Wood KE. Outcome of unoperated discogram-positive low back pain. Spine (Phila Pa 1976). 1995;20(18):1997-2000; discussion 2000-1991.

10. Deyo RA, Gray DT, Kreuter W, Mirza S, Martin BI. United States trends in lumbar fusion surgery for degenerative conditions. Spine (Phila Pa 1976). 2005;30(12):1441-1445; discussion 1446-1447.

11. Talia AJ, Wong ML, Lau HC, Kaye AH. Comparison of the different surgical approaches for lumbar interbody fusion. J Clin Neuroscience. 2015;22:243-251.

12. Rihn JA, Patel R, Makda J, et al. Complications associated with single-level transforaminal lumbar interbody fusion. Spine J. 2009;9(8):623-629.

13. Peng CW, Yue WM, Poh SY, Yeo W, Tan SB. Clinical and radiological outcomes of minimally invasive versus open transforaminal lumbar interbody fusion. Spine (Phila Pa 1976). 2009;34(13):1385-1389.

14. Oxland TR, Grant JP, Dvorak MF, Fisher CG. Effects of endplate removal on the structural properties of the lower lumbar vertebral bodies. Spine (Phila Pa 1976). 2003;28(8):771-777.

15. Lowe TG, Hashim S, Wilson LA, et al. A biomechanical study of regional endplate strength and cage morphology as it relates to structural interbody support. Spine (Phila Pa 1976). 2004;29(21): 2389-2394.

16. Tan JS, Bailey CS, Dvorak MF, Fisher CG, Oxland TR. Interbody device shape and size are important to strengthen the vertebra-implant interface. Spine (Phila Pa 1976). 2005;30(6):638-644.

17. Voronov LI, Conti Mica M, Carandang G, Havey RM, Wojewnik B, Patwardhan AG. Biomechanics of expandable lumbar interbody fusion cage deployed through transforaminal approach. Presented at: the 32nd Annual Meeting of the Section on Disorders of the Spine and Peripheral Nerves (Spine Summit 2016); March 19; 2016; Orlando, FL.

18. Copay AG, Glassman SD, Subach BR, Schuler BS, Carreon LY. Minimum clinically important difference in lumbar spine surgery patients: a choice of methods using the Oswestry Disability Index, Medical Outcomes Study questionnaire Short Form 36, and pain scales. Spine J. 2008;8(6):968-974.

19. Hou Y, Yuan W. Influences of disc degeneration and bone mineral density on the structural properties of lumbar end plates. Spine J. 2012;12(3):249-256.

20. Fukuta S, Miyamoto K, Hosoe H, Shimizu K. Kidney-type intervertebral spacers should be located anteriorly in cantilever transforaminal lumbar interbody fusion: analyses of risk factors for spacer subsidence for a minimum of 2 years. J Spinal Disord Tech. 2011;24(3):189-195.
Medical Devices: Evidence and Research

\section{Publish your work in this journal}

Medical Devices: Evidence and Research is an international, peerreviewed, open access journal that focuses on the evidence, technology, research, and expert opinion supporting the use and application of medical devices in the diagnosis, monitoring, treatment and management of clinical conditions and physiological processes. The identification of novel
Dovepress

devices and optimal use of existing devices which will lead to improved clinical outcomes and more effective patient management and safety is a key feature. The manuscript management system is completely online and includes a quick and fair peer-review system. Visit http://www. dovepress.com/testimonials.php to read real quotes from authors. 